\title{
Synthese von 1,3,5-Triazinen aus Aminosäure-Derivaten (III)
}

\author{
Eiichi Kuwano, Eiji Taniguchi und Kazuyuki MaEkawa \\ Landwirtschaftliche Fakültät, Kyushu Universität
}

Eingegangen September 19, 1972

\begin{abstract}
Amino acid esters as well as peptideesters reacted directly, without addition of DCC, with dimethylbiguanide to give triazine-derivatives. The yields of the triazine-derivatives were fairly good and the racemization did not occur during the reactions. The carbobenzoxy group of $\mathrm{N}$-end was removed by treatment with $25 \% \mathrm{HBr}$ at room temp. without decomposition of the triazine-ring formed at the C-terminal.
\end{abstract}

Wenn Triazin-Derivate von N-geschützten Aminosäuren bzw. Peptiden durch Reaktion mit S-Methyl-guanylisothiuronium-methosulfat, Dimethylbiguanid oder Morpholinobiguanid synthetisiert wurden, war die Ausbeute nicht genügend. ${ }^{1,2)}$ Die hier beschriebenen Versuche wurden unternommen, um die Carboxy-Gruppe von instabilen Aminosäuren in den Ring einzuschliessen, was bis heute noch nicht gelungen war, und um die Ausbeute zu verbessern.

Die Folge davon ist, dass die Ester der Aminosäuren verhältnissmässig leicht bei niederer Temperatur und unter Abwesenheit von einem Kondensierungsmittel wie DCC bis zu einem befriedigenden Grade reagieren. Ferner findet wegen der Abwesenheit von DCC keine Razemizierung statt.

Die Esterifizierung von Aminosäuren wurde sowohl nach gewöhnlicher Weise ${ }^{3}$ als auch mittels aktivierter Ester-Methode durchgeführt, welche durch Verknüpfung mit 1-Hydroxybenzotriazol ${ }^{7}$ oder 3-Hydroxyisoxazol erreicht wurde. Jene Esterifizierungs-Stufe war nicht quantitativ, aber der Ring-Schluss kam mit guter Ausbeute zustande (Fig. 1).

$\mathrm{Da}$ die Aminosäuren in wässriger Lösung mit Meerweinschem Reagens ${ }^{4}$ unter milden Bedingungen reagieren und Ester ergeben, ${ }^{5,6}$ ist dieser Umweg wahrscheinlich ein geeignetes

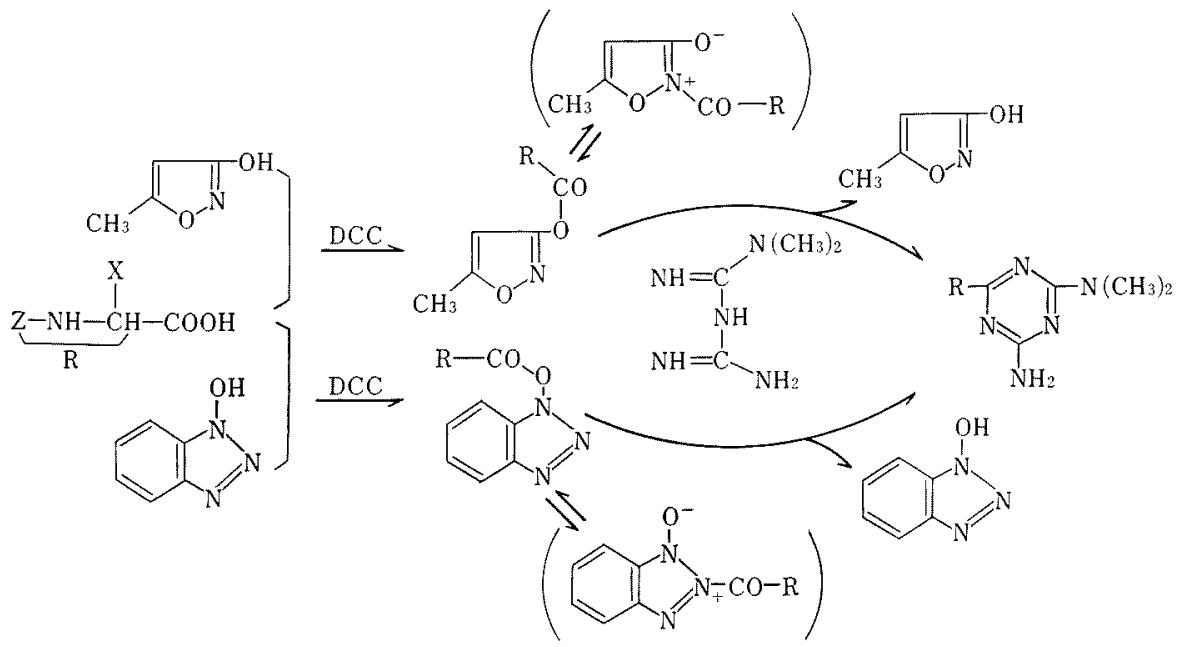

FIG. 1. Synthese von Triazin-Derivaten aus Aminosäuren mittels aktivierter Ester-Methode.

Abkürzungen, Bz: Benzoyl-, Ts-: Tosyl-, Z-: Carbobenzoxy- Nap-: NaphthylDMGG: Dimethyl-biguanid, DCC: Dicyclohexyl-carbodiimid 
Verfahren, um die Carboxylgruppe von komplizierten oder instabilen Aminosäuren in den Triazin-Ring einzuschliessen.

Dieses Verfahren wurde im Gegensatz zu den früher beschriebenen Methoden bei Peptiden mit befriedigender Ausbeute vollzogen.

Die Ergebnisse über Thr, Ser, Gln, Asn, Cys und His wurden hinzugefügt, die früher nicht behandelt werden konnten.

Schliesslich konnte man durch dieses sowie durch früher beschriebene Verfahren die Carboxylgruppe von Aminosäuren alle in einen Triazin Ring einschliessen. Die Ergebnisse werden in Tafel 1 zusammengestellt.

Die IR-Spektren von erneut gebildeten Triazin-Derivaten von Amino-säuren werden in Fig. 2, 3 gegeben. Dies zeigt die auf den Triazin-Ring zurückführbaren Absorptionen bei $1540 \mathrm{~cm}^{-1}$ und $815 \mathrm{~cm}^{-1}$, wie schon früher berichtet wurde. ${ }^{1,2)}$ Nach Einbau in den

TAFEL I. VERSChIEDENER EINBAU-WEg DER CARBOXYL-GRUPPEN VON AMINOSÄUREN") IN DEN TRIAZIN-RING

\begin{tabular}{|c|c|c|c|}
\hline $\mathrm{NH}_{2}$-Gruppe & Carboxyl-Gruppe & Abgeleitete Triazin-Derivate & Ref. \\
\hline Geschützte & freie & 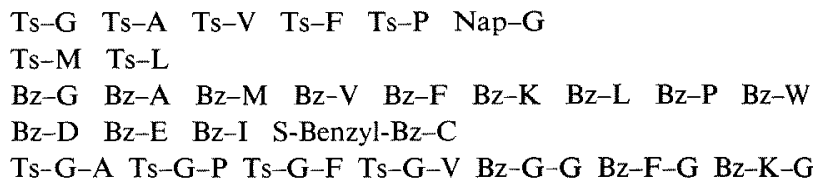 & $\begin{array}{l}1 \\
2 \\
2\end{array}$ \\
\hline Freie & Ester & 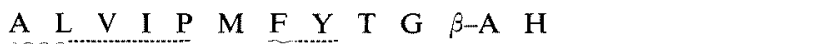 & \\
\hline Geschützte & Ester & 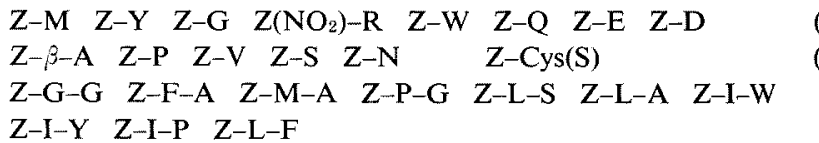 & (t) \\
\hline
\end{tabular}

\#) Aminosäuren wurden mit einem Schriftzeichen ausgedrückt. ${ }^{91}$

(t) ... wurde nach Zusatz von 1-Hydroxybenzotriazol behandelt.

(o) . . w wurde nach Zusatz von 3-Hydroxy-5-methylisoxazol behandelt.

mit guter Ausbeute, ------ ohne Razemizierung.

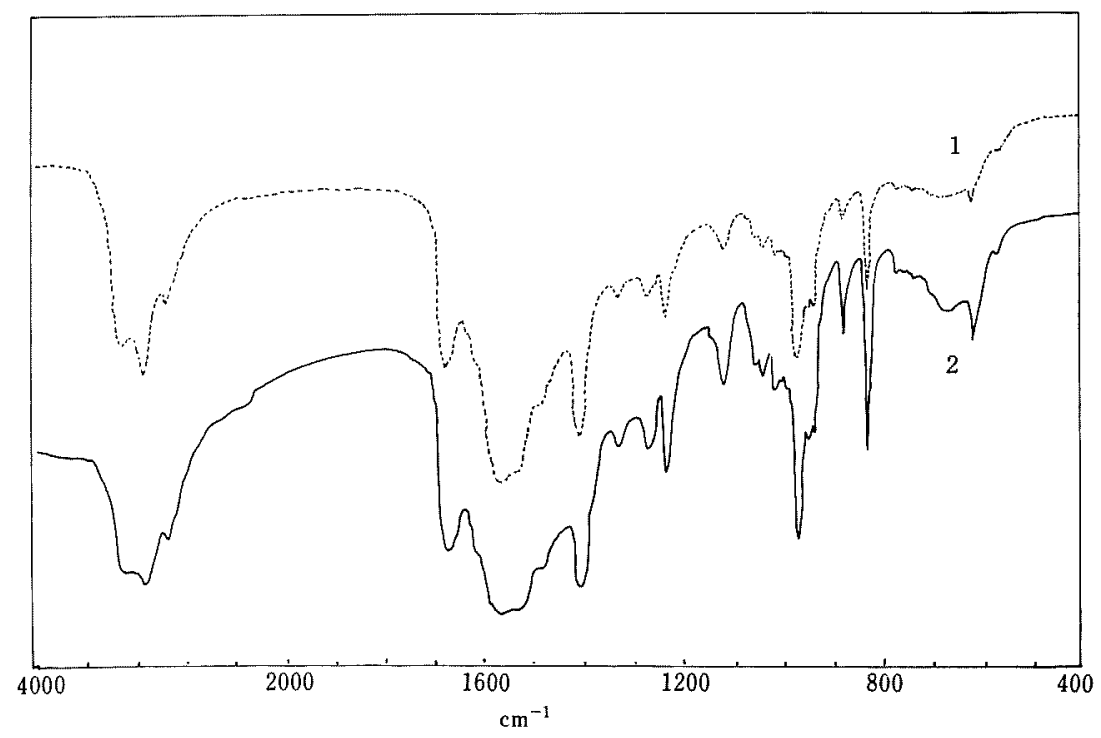

FIG. 2. IR-Spektren von den aus Methionin erhaltenen s-Triazin-Derivat.

1. Z-Gruppe wurde aus dem Z-Methionin-Derivat mittels $\mathrm{HBr}$ entfernt

2. Wurde durch Ester-Methode dargestellt. 


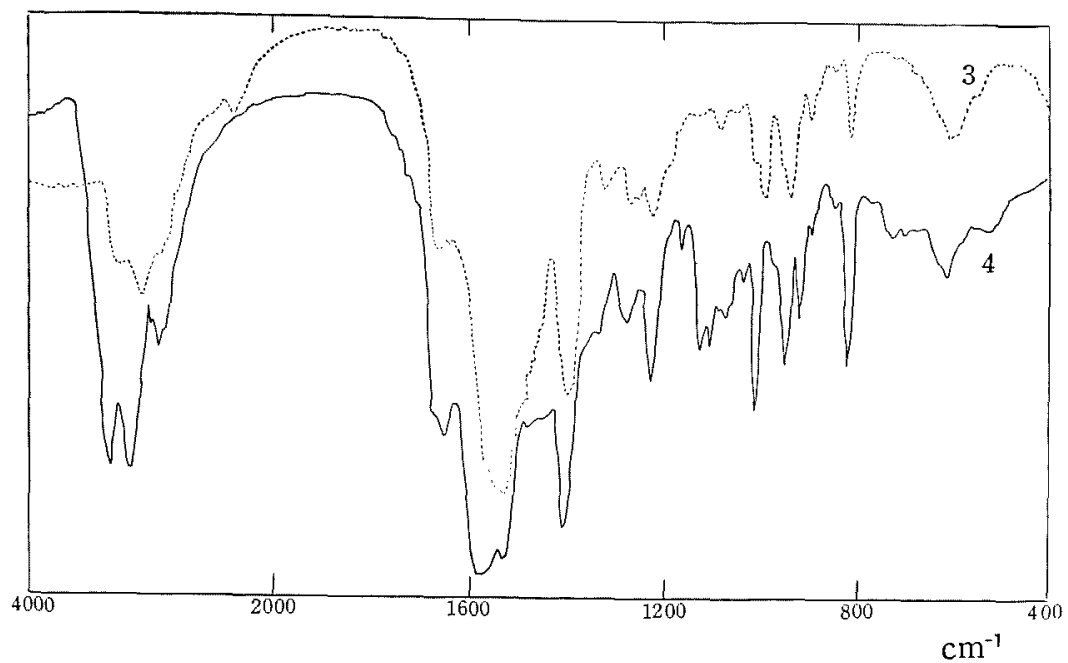

FIG. 3. IR-Spektren von den aus Ala und Thr erhaltenen s-Triazin-Derivat. 3. aus Ala, 4. aus Thr

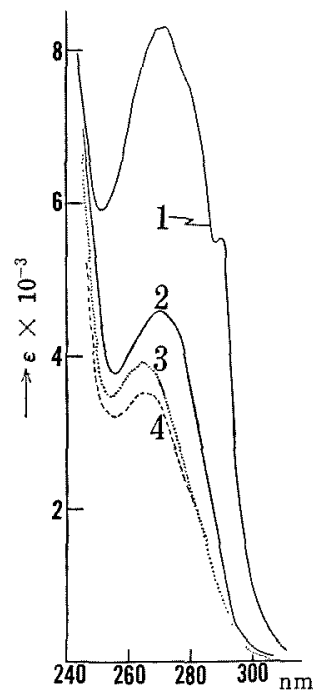

FIG. 4. UV-Absorptionsspektren von den aus Cbz-Trp, Tyr, Phe und Ala erhaltenen TriazinDerivaten (Lösungsmittel: $\mathrm{MeOH}$ ).

1. aus Cbz-Trp $(\varepsilon=8,310)$, 2. aus Tyr $(\varepsilon=4,600)$

3. aus Phe $(\varepsilon=3,900)$,

4. aus Ala $(\varepsilon=3,500)$

Triazin-Ring verschob jede der Amino-säuren ihren Drehsinn um $20^{\circ}$ nach links, abgesehen von Triazin-Derivaten des $\mathrm{Tyr}\left(20^{\circ}\right.$ nach recths). Die UV-Absorptions Spektren von Triazin-Derivaten von Trp, Tyr, Phe und Ala werden in Fig. 4 gegeben.

Die Carbobenzoxy-Gruppe vom AminoEnde, die in diesem sowie früheren Berichten gelegentlich gebraucht wurde, wurde zuletzt leicht Stehenlassen über Nacht mit $25 \% \mathrm{HBr}$ bei Raumtemp.entfernt.

Über die physiologischen Wirkungen von den erhaltenen Derivaten wird anderswo berichtet werden.

\section{BESCHREIBUNG DER VERSUCHE}

\section{Darstellung von Aminosäureestern und Pep-} tidestern

a) Methyl-, oder Äthylester. Aminosäuren wurden in Methanol bzw. Äthanol unter Zusatz von Thionylchlorid esterifiziert. ${ }^{3)}$ Der erhaltene Ester wurde aus demselben Alkohol und Äther umkristallisiert. Ölige Produkte wurden nach Trocknung in einem $\mathrm{NaOH}$-Exsiccator ohne weitere Reinigung gebraucht.

b) Darstellung der aktiven Ester mittels 1-Hydroxybenzotriazol. ${ }^{7)}$ (i), Z-Met $(3.5 \mathrm{~m}$ $\mathrm{Mol}$ ) und 1-Hydroxybenzotriazol (4.4 m Mol) wurden in $30 \mathrm{ml}$ Äthanol aufgelöst, und dazu Dicyclohexylcarbodiimid $(3.9 \mathrm{~m} \mathrm{Mol})$ zugesetzt. Das Rühren wurde 1 Stunde lang bei $0^{\circ}$, dann 3 Stunden lang bei Raumtemp. fortgesetzt, um Ester von Benzotriazol darzustellen. Der Ester wurde ohne Isolierung unmittelbar zur Reaktion mit DMGG benutzt. 


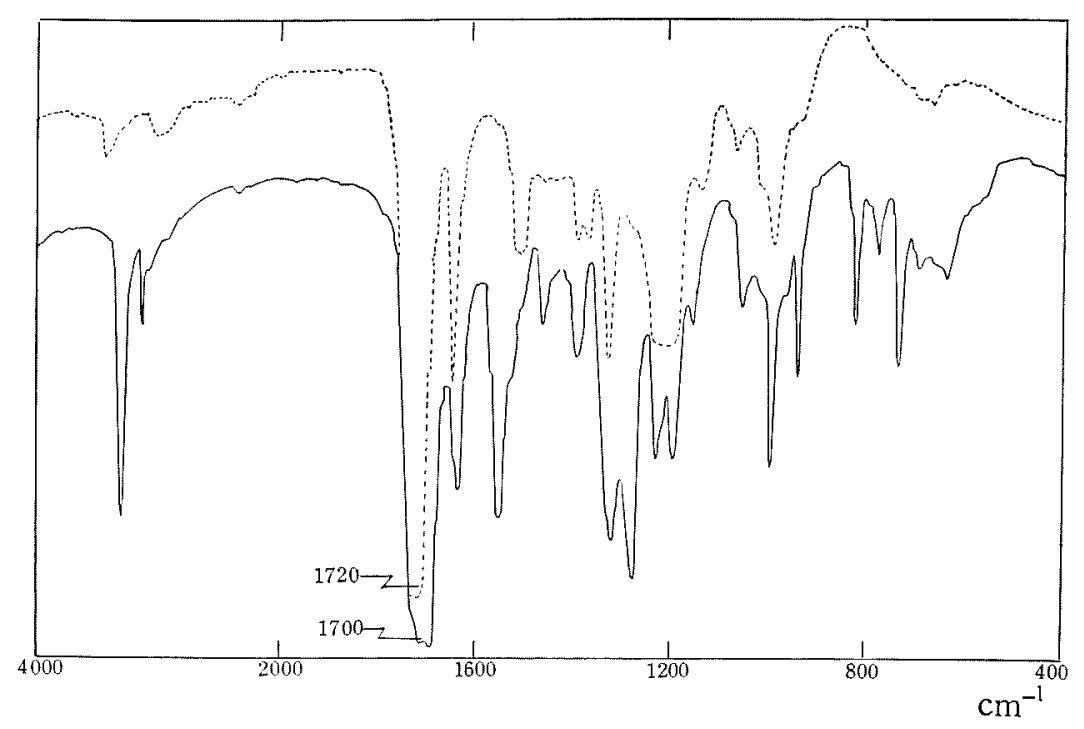

FIG. 5. IR-Spektren von Z- $\beta$-Ala-[3-hydroxy-5-methylisoxazolester].

- $\mathrm{KBr}$-Pressling, --- Lösung in $\mathrm{CHCl}_{3}$

(ii), Z-Glu und Z-Gln standen nach diesem Verfahren zur Verfügung.

c). Anwendung von 3-Hydroxy-5-methylisoxazol für Darstellung aktiver Ester. (i), Z- $\beta$-Ala(1 m Mol) und 3-Hydroxy-5-methylisoxazol $^{8)}(1.2 \mathrm{~m} \mathrm{Mol})$ wurden in $30 \mathrm{ml}$ Tetrahydrofuran aufgelöst und auf $0^{\circ}$ gekühlt. Unter Rühren wurde DCC (1.2 m Mol) zugesetzt. Es wurde 3 Stunden lang bei Raumtemp.gerührt und dann, nachdem Dicyclohexylharnstoff entfernt worden war, auf ein kleines Volumen eingeengt. Nach Zusatz von $30 \mathrm{ml}$ Äther entstand ein farbloser kristallinier Niederschlag. Der Niederschlag wurde aus Äthanol umkristallisiert. Ausbeute $73 \%$.

$$
\mathrm{C}_{15} \mathrm{H}_{16} \mathrm{O}_{5} \mathrm{~N}_{2} \text { : }
$$

Ber. C, $59.20 \% \mathrm{H}, 5.30 \% \mathrm{~N}, 9.21 \%$ (304) Gef. C, $59.09 \%$ H, 5.43\% N, 9.20\% Die IR-Spektren von dem aktivierten Ester wurden in Fig. 5 gezeigt. (ii), Z-Ser, Z-P, $\mathrm{Z}-\mathrm{V}$ und $\mathrm{Z}-\mathrm{Asn}$ wurden nach diesem Verfahren behandelt.

II. Darstellung von Triazin-Derivaten aus Aminosäureestern bzw. N-geschützten Peptidestern.
(A) Aus Aminosäureestern

a-1), Aus Methyl-oder Äthylestern.

Phenylalanin-methylester wird als Beispiel angeführt. Der aus $4 \mathrm{~g}$ Phe dargestllte Phemethylester wurde in $50 \mathrm{ml} \mathrm{MeOH}$ aufgelöst und dann mit $10 \mathrm{~g}$ DMGG unter Rühren bei $0^{\circ}$ versetzt. Nachdem 2 Tage lang bei Raumtemp.gerührt worden war, wurde das abgeschiedene DMGG.HCl Salz filtriert. Nach Entfernen des Lösungsmittels wurde der Rückstand mit $\mathrm{CHCl}_{3}$ extrahiert. Die $\mathrm{CHCl}_{3}$ Lösung wurde mit einer geringen Menge Wasser gewaschen, dann mit $\mathrm{Na}_{2} \mathrm{SO}_{4}$ entwässert, und zur Trocknung eingeengt. Der Rückstand wurde aus Benzol umkristallisiert. Schmelzpt. $127 \sim 8^{\circ}$, Ausbeute $4 \mathrm{~g}(63 \%$ aus Phe).

Ala-Et-Ester, Leu-Et-, Val-Me-, Ile-Et-, Pro-Me, $\beta$-Ala-Et-, Met-Me-, Tyr-Et-, ThrMe- usw. wurden jede für sich auf ähnliche Weise wie Phe ohne vorhergehende N-Blockierung unmittelbar mit DMGG bei $0^{\circ}$ Raumtemp.behandelt.

a-2), Der mit Benzylgruppe SH-geschützten Cys $(H)$ wurde angewendet, um mit DMGG zu kondensieren, während Z-Cys (S) unmittelbar esterifiziert wurde. 
b), Aus dem aktiven Ester von 1-Hydroxybenzotriazol Z-Glu-[1-hydroxybenzotriazoldiester], das aus Z-Glu (3 g) und 1-Hydroxybenzotriazol $(3.4 \mathrm{~g})$ in Gegenwart von DCC $(4.8 \mathrm{~g})$ dargestellt wurde, wurde mit DMGG $(7 \mathrm{~g})$ in methanolischer Lösung unter Rühren bei $0^{\circ}$ versetzt. Nachdem 1 Tag lang bei Raumtemp. gerührt worden war, wurde der abgeschiedene Niederschlag filtriert. Nach Entfernen des Lösungsmittels wurde der Rückstand mit $\mathrm{CHCl}_{3}$ extrahiert. Die $\mathrm{CHCl}_{3}$ Lösung wurde mit einer geringen Wasser gewaschen, dann mit $\mathrm{Na}_{2} \mathrm{SO}_{4}$ entwässert, und zur Trockne eingeengt. Der Rückstand wurde aus Äthanol umkristallisiert. Schmelzpt. $118 \sim 120^{\circ}$, Ausbeute $3.3 \mathrm{~g}(66 \%)$.

Die Carboxylgruppe von Z-Arg $\left(\mathrm{NO}_{2}\right)$, di-Z-Lys und Z-Gln wurde auf ähnliche Weise im Triazin-Ring eingeschlossen.

b-2), Di-Z-Tyr-Ester verlor bei der Kondensation mit DMGG seine Schütz-Gruppe von Phenol-OH.

c), Aus dem aktiven Ester von 3-Hydroxy-5methylisoxazol

Z-Ser-[3-hydroxy-5-methylisoxazolester], das aus Z-Ser (2.4 g) und 3-Hydroxy-5-methylisoxazol $(1 \mathrm{~g})$ in Gegenwart von DCC dargestellt wurde, wurde in $15 \mathrm{ml} \mathrm{MeOH}$ aufgelöst und dann mit $3 \mathrm{~g}$ DMGG unter Rühren bei $0^{\circ}$ versetzt.

Es wurde über Nacht bei Raumtemp.gerührt und dann auf ein kleines Volumen eingeengt. Nach Zusatz von etwa $30 \mathrm{ml}$ Wasser entstand ein farbloser Niederschlag. Die Umkristallisierung wurde 2 mal aus Methanol durchgeführt. Schmelzpt. 154 $6^{\circ}$, Ausbeute $\operatorname{g~}(30 \%)$.

\section{(B). Darstellung von Triazin-Derivaten aus}

\section{Peptidestern.}

Z-Glycylglycinethylester $(0.2 \mathrm{~g})$ wurde in $10 \mathrm{ml}$ Äthanol aufgelöst und mit $0.3 \mathrm{~g} \mathrm{DMGG}$ versetzt. Es wurde 2 Tage lang bei Raumtemp. gerührt und dann im Rotationsverdampfer auf ein kleines Volumen eingeengt. Nach Zusatz von $30 \mathrm{ml}$ Wasser entstand ein farbloser Kristall. Die Umkristallisierung wurde aus Methanol durchgeführt. Schmelzpt. 179 $180^{\circ}$. Ausbeute $83 \%$.
Andere Peptidester wurden auf ähnliche Weise behandelt. In einigen Fällen wurde zwecks Reinigung Säulen-Chromatographie verwendet. Die dabei erfundenen Resultate werden in Tafel 2 zusammengestellt.

\section{Dünnschicht-Chromatographie und Reinig- ung mittels Säulen-Chromatographie.}

a) Kieselgel Chromatographie. Die methanolische Lösung, ausgehend von $2.4 \mathrm{~g}$ Z-Met-Ala-Et-Ester und 2.8 g DMGG, wurde auf eine Kieselgel-Säule $(150 \times 28 \mathrm{~mm})$ aufgetragen. Die Elution erfolgte mit $100 \mathrm{ml}$ Benzol, $150 \mathrm{ml}$ Benzol: AcOEt (2:1) und $100 \mathrm{ml}$ desselben $(1: 1)$, das Eluat wurde in 5 ml-Fraktionen gesammelt. Die letzter Fraktion enthielt $600 \mathrm{mg}$ Z-Met-Ala-TriazinDerivat.

b) Amberlite IRC-50 Säulen-Chromatographie. Die methanolische Reaktionslösung ausgehend von $1 \mathrm{~g}$ Glycinmethylester und $1 \mathrm{~g}$ DMGG, wurde zur Trockne eingedampft. Nach Zusatz von $\mathrm{HCl}$ wurde das abgeschiedene DMGG-HCl Salz abfiltriert. Das Filtrat wurde mit Ammoniak neutralisiert, und auf ein kleines Volumen eingeengt, und dann auf eine Amberlite IRC-50 $\mathrm{H}^{+}$-Säule $(350 \times 40 \mathrm{~mm})$ aufgetragen. Die Elution erfolgte zunächst mit Wasser, dann $1 \mathrm{~N}$ wässriger Ammoniaklösung. Die letzte Fraktion wurde gesammelt. Sie enthielt $450 \mathrm{mg}$ Gly-TriazinDerivat.

c) Dünnschicht-Chromatographie (TLC).

In TLC wurde Kieselgel als Träger verwendet. Als Lösungsmittel wurde $\mathrm{BuOH}$ : AcOH: Wasser $=12: 3: 5$ benutzt. Der Nachweiss der Flecken erfolgte durch Behandlung mit Jod bzw. Ninhydrin.

\section{Beseitigung N-geschützter Gruppen}

a), Zum Z-Ala-Triazin-Derivat ( $0.3 \mathrm{~g}$ ) wurde $1 \mathrm{ml} 25 \% \mathrm{HBr}$ Essigsäurelösung zugesetzt. Das Gemisch wurde bei Raumtemp.über Nacht stehen gelassen. Nach Zusatz von $20 \mathrm{ml}$ Äther entstanden farbloser Kristall. Das Lösungsmittel wurde nun dekantiert, der Kristall mit Äther 3 mal durchgewaschen. 


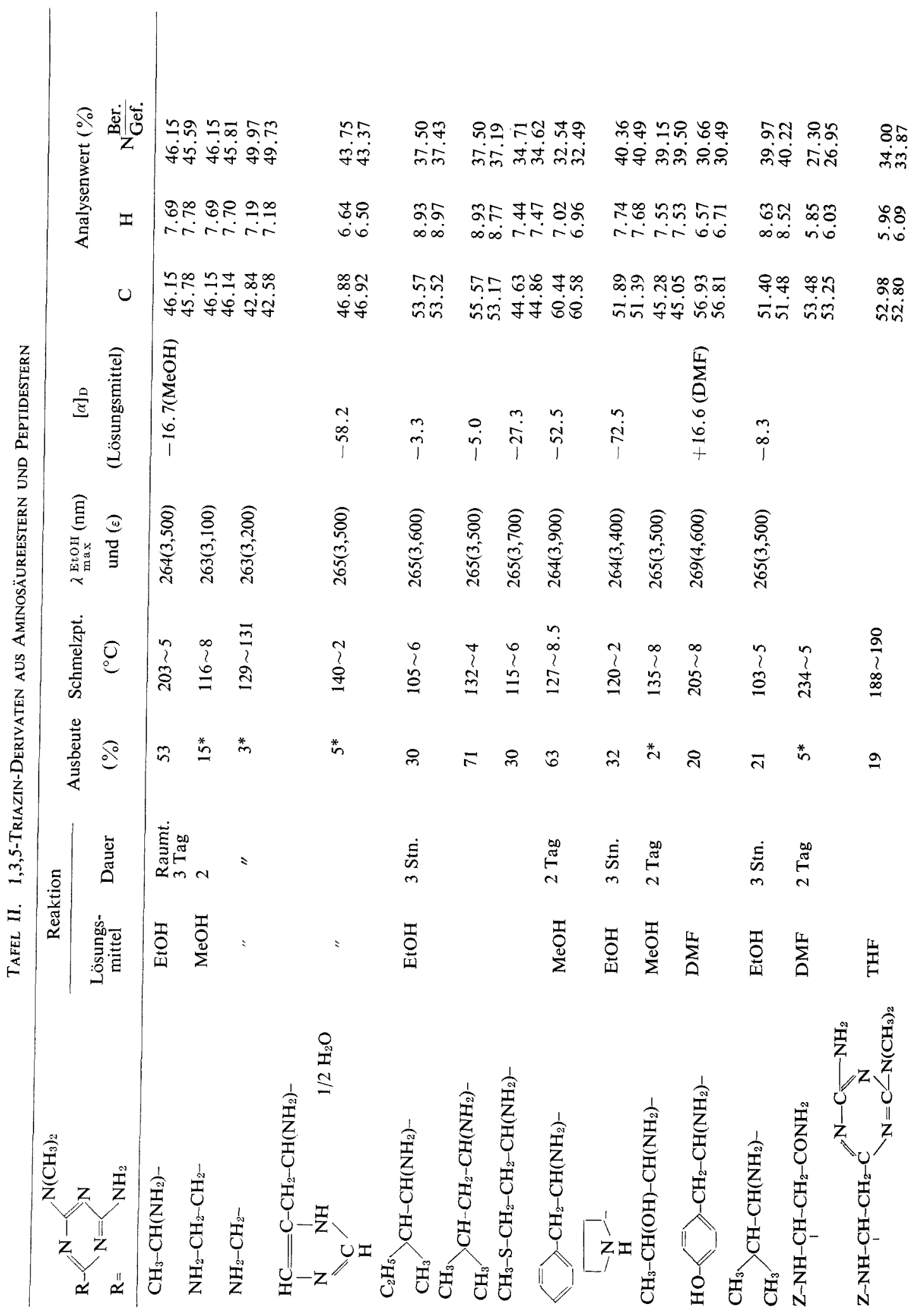


Synthese von 1,3,5-Triazinen aus Aminosäure-Derivaten (III)

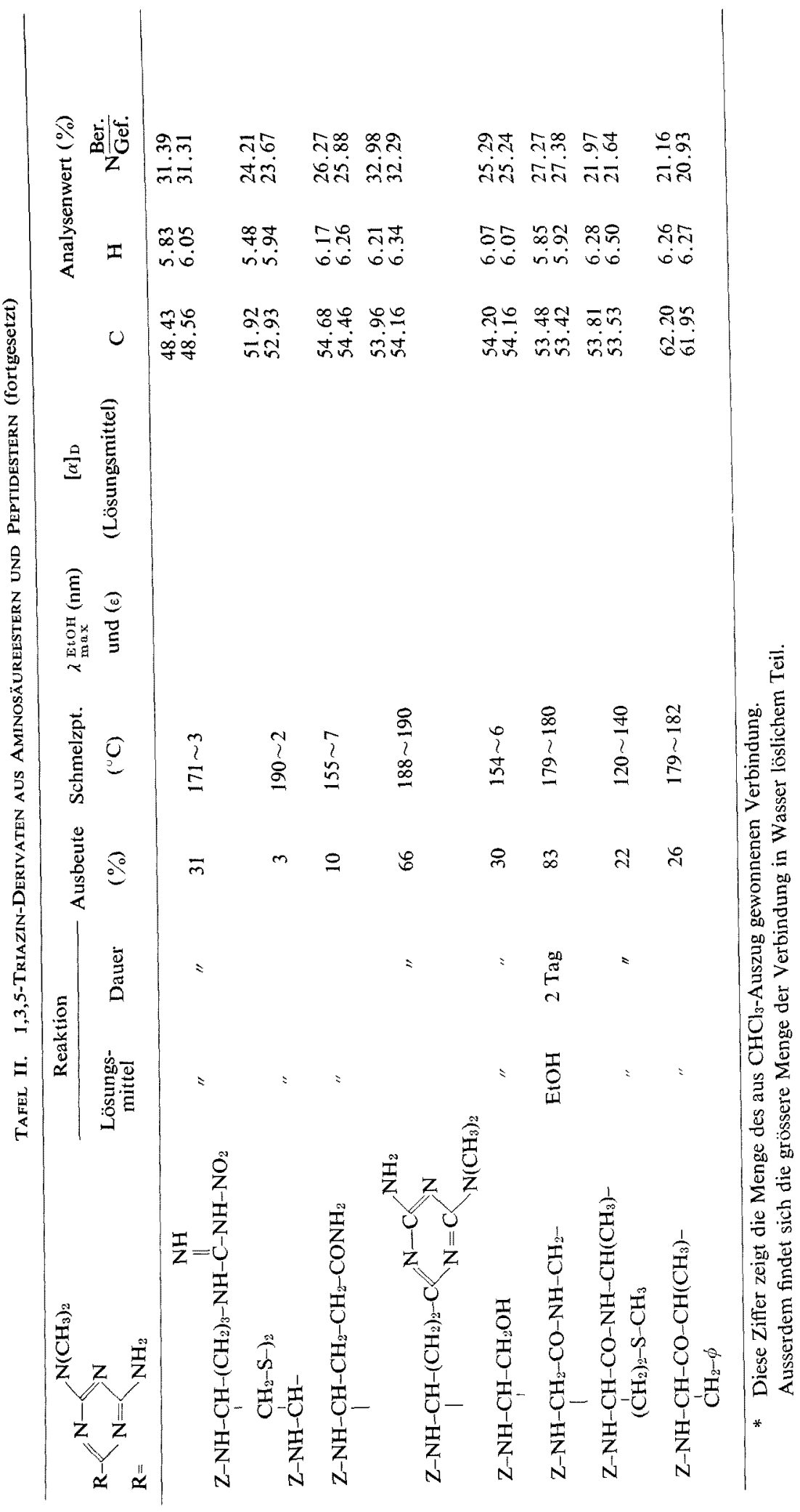


Ausbeute $0.2 \mathrm{~g}(60 \%)$. Die Umkristallisierung wurde aus Äthanol und Äther vollgezogen.

$\mathrm{C}_{7} \mathrm{H}_{16} \mathrm{~N}_{6} \mathrm{Br}_{2}$ :

(344) Ber. C $24.42 \% \mathrm{H} 4.65 \%$ N $24.42 \%$

$$
\text { Gef. } 24.49 \quad 4.78 \quad 24.22
$$

b), Z-Phe-Triazin-Derivat, Z-Tyr- und ZGly-Gly- wurden in ähnlicher Weise wie oben behandelt. Ausbeuten waren je 70, 75 und $30 \%$. Weil das so erhaltene Gly-Gly-Triazin. $\mathrm{HBr}$-Salz im Gegensatz zu anderen Verbindungen hygroskopisch war, wurde es durch Zusatz von $25 \% \quad \mathrm{NH}_{4} \mathrm{OH}$ freigesetzt. Der dabei ausgeschiedene Niederschlag wurde aus Äthanol umkristallisiert.Schmelzpt.204 $\sim 6^{\circ}$

Phe-Triazin-Derivat: $\mathrm{C}_{13} \mathrm{H}_{20} \mathrm{~N}_{6} \mathrm{Br}_{2} \quad$ (420)

$$
\mathrm{C} \% \quad \mathrm{H} \% \quad \mathrm{~N} \%
$$

$\begin{array}{llll}\text { Ber. } & 37.14 & 4.76 & 20.00\end{array}$

Gef. $\quad 37.29 \quad 4.86 \quad 19.55$

Tyr-Triazin-Derivat: $\mathrm{C}_{13} \mathrm{H}_{20} \mathrm{ON}_{6} \mathrm{Br}_{2}$

$\begin{array}{llll}\text { Ber. } & 35.78 & 4.59 & 19.27\end{array}$

Gef. $\quad 36.01 \quad 4.83 \quad 18.83$

Gly-Gly-Triazin-Derivat: $\mathrm{C}_{8} \mathrm{H}_{15} \mathrm{ON}_{7} \quad$ (255)

$\begin{array}{rrrr} & \mathrm{C} \% & \mathrm{H} \% & \mathrm{~N} \% \\ \text { Ber. } & 42.67 & 6.67 & 43.56 \\ \text { Gef. } & 42.55 & 6.53 & 42.74\end{array}$

\section{LITERATUR}

1) E. Kuwano, E. Taniguchi und K. Maekaw: Agr. Biol. Chem., 35, 1572 (1971).

2) E. Kuwano, E. Taniguchi und K. Maekawc ibid., 35, 1759 (1971).

3) J. Bello, Biochim. Biophys. Acta, 20, 427 (1956,

4) H. Meerwein, Org. Syn., 46, 113 (1966).

5) S. M. Parsons, L. Jao, F. W. Dahlquist, C. I Borders, T. Groff und J. Racs, Biochem., 8, 70 (1969).

6) O. Yonemitsu, T. Hamada und Y. Kanaoka Tetrahedron Letters, 23, 1819 (1969).

7) K. König und R. Geiger, Chem. Ber., 103, 78 (1970).

8) K. Tomita, M. Nagano, T. Yanai, H. Oka, T Murakami und N. Sampei, Ann. Sankyo Res Lab., 22, 215 (1970).

9) IUPAC-IUB Commission on Biochemical No menclature; Biochemistry, 7, 2703 (1968). 